\title{
Il est bien temps, tiens, de faire des voeux pour 2014...
}

\author{
Y. Panis \\ C Springer-Verlag France 2014
}

A l'heure où j'écris ce modeste éditorial pour Colon \& Rectum, notre revue bien aimée, sur ma Remington numéro 7 (modèle de 1887), je me prends à rêver tout haut de ce que pourrait être notre beau métier de chirurgien colorectal en 2014.

Que pouvons-nous espérer pour l'année 2014?

Un meilleur salaire ? Aucune chance. Une voiture de fonction payée par l'APHP ? Non plus...Un bureau où le chauffage marche? N'exagérons rien! Une SNFGE qui se souvient qu'il y a bien longtemps (à l'époque Jérémie Lefevre n'était même pas né et Guillaume Meurette était en CM2, c'est dire...), trainaient dans les couloirs des JFHOD pleins de chirurgiens heureux d'être là, dont votre serviteur? $\mathrm{Ne}$ rêvons pas. Là on nage en pleine science fiction.

Donc, il va falloir être plus modeste j'ai l'impression. Réfléchissons alors un peu sur l'avenir de nos chères Sociétés Savantes. En effet, toujours à l'époque où Guillaume Meurette passait avec difficulté le certificat d'étude (séchant sur la couleur du cheval blanc d'Henri IV), votre serviteur, toujours lui (j'aime bien parler de moi...) était aux responsabilités à la SFCD, et déjà membre du conseil d'administration de la SNFCP. J'ai même connu et tutoyé Emmanuel Tiret. C'est dire si je suis vieux...Bref, je m'égare. Donc, le fonctionnement des Sociétés Savantes, je me targue de m'y connaître un peu. Ou plutôt, je dois dire, je m'y connaissais ! Car le monde a bien changé depuis 20 ans. Le monde déjà tout court évidemment, mais aussi et surtout celui de nos sociétés savantes (SS en abrégé c'est plus simple pour la suite du texte).

Bref rappel en arrière. De quoi causait-on à la fin du dernier millénaire et au tout début de l'actuel ? On était dans des sociétés savantes, et donc on causait de science. CQFD. Et aussi de basse politique évidemment. Mais au moins cela

\section{Y. Panis $(\bowtie)$}

Service de Chirurgie Colorectale,

Pôle des Maladies de 1'Appareil Digestif,

Hôpital Beaujon - Assistance Publique des Hôpitaux

de Paris (APHP), Université Paris VII (Denis Diderot),

100, boulevard du Général Leclerc, 92118 Clichy cedex

e-mail : yves.panis@bjn.aphp.fr avait un avantage : on comprenait tous les mots. Et on pouvait se battre à armes égales pour défendre ses intérêts où ceux de son « clan ». Aujourd'hui que nenni ! Tout ceci est bien fini! Ouste! Adieu nos chères engueulades! Aujourd'hui on cause DPC (LE DPC, pour les ignares, et pas LA DPC, opération emblématique du chirurgien « qui en a »), la FSM et les CNP, dont le CNP-HGE forcément, voire la FSMAD (je sais pas ce que c'est, désolé), et la Fédération des Spécialités (il paraît qu'il y en a une pour la chirurgie digestive), Accréditation, etc, etc. Il paraît même que « le CEFA HGE contrôle le DPC ». C'est dingue! Mais je comprends toujours rien... Avec l'idée de base énoncée à chaque réunion : "attention mon gars, tu as tort de ne pas t'y intéresser! Tu es foutu ! ça va devenir obligatoire! Tu es mort! » Bref, on vous prédit l'enfer si vous ne suivez pas le chemin tout tracé par nos SS.

Et moi dans mon coin je me disais quoi dans ces réunions ? D'abord je suis passé par une phase d'incompréhension totale des débats (« mais de quoi ils causent ? « « Finalement, ils ont peut-être raison : les chirurgiens doivent être moins intelligents que les médecins car je n'y comprends rien ! » etc, etc.). Ensuite, évidemment, car c'est mon tempérament, j’ai réagi : " ah ah, je me marre ! Déjà il y a 20 ans, quand sous Kouchner (si j'ose dire) on causait de FMC et qu'il fallait avoir ses points, et que c'était obligatoire, et bien finalement il ne s'est rien passé. Alors comme il n'y a pas d'argent, et bien ce coup-ci ça sera pareil, et toc ! ». Mais bon, faut être honnête, dans nos conseils d'administration, je suis ultra-minoritaire je pense. Il n'y a qu'à voir les débats enflammés sur le sujet pour se rendre compte que mes collègues suivent de près le débat. Qu'ils sont au jus. Ou alors ça ennuie tout le monde, mais personne n'ose rien dire ? Pas impossible finalement. Et ça serait plutôt rassurant...

Je vais vous faire une confidence : tout ceci m'emmerde. Désolé pour la vulgarité. D'abord parce que je n'y comprends rien. Ensuite parce que cela ne m'intéresse pas mais alors vraiment pas du tout. Enfin et surtout, et c'est l'objet (un peu tardif, je reconnais) de mon éditorial qui n'a ni queue ni tête : à mes yeux, une société savante ne devrait causer que de RPC, Conférence de consensus, organisation du programme scientifique du congrès annuel, fiches d'informations et c'est 
tout ! Le reste devrait être discuté ailleurs.... ou sans moi. Voilà, c'est dit. Je sens que je vais pas me faire un paquet d'amis mais bon tant pis. Ce n'est pas notre boulot. Je veux bien que les syndicats et les Fédérations s'en occupent. Très bien. Mais pas nous. Ou pas moi.
Donc, en conclusion, mon vœu pour 2014 ? Avoir à nouveau de belles sociétés savantes où tout le monde s'aime et où on cause de médecine, de chirurgie, de proctologie, bref de belles choses...

Bonne année à tous ! 